\title{
Moving Away From Anecdotal Responses to Questions Faculty Have Concerning Broader Impacts
}

\author{
Michael Thompson ${ }^{1}$ \\ ${ }^{1}$ University of Oklahoma
}

May 5, 2020

\begin{abstract}
Many faculty researchers, University administrators, proposal development individuals and organizations, engagement specialists, Societal Benefit Organizations (SBOs), and Societal Benefit Professionals (SBPs) have asked and indicated the need for a researched evidence-based response to the following question: What is one of the most important keys to developing broader impacts for the National Science Foundation (NSF)? This question has become especially salient for faculty submitting proposals to NSF or other agencies, foundations, and organizations with different types of broader impacts foci. Faculty know it is vital to develop broader impacts, but they do not necessarily know what the deeper meaning of broader impacts is. To truly understand the scope of broader impacts, we need to go beyond anecdotal descriptions of what others have done. This article introduces a research-based framework for understanding, practicing, and starting to operate in a broader impacts paradigm. This response is a brief synopsis based off several works-in-progress that either have been or will be submitted for publication in peer-reviewed journals.
\end{abstract}

\section{Brief Introduction, Background, and Methodology}

Many do not realize that the concept, meaning, and methodology of broader impacts represent an international phenomenon. An investigation into this phenomenon revealed that at least eighty-two percent $(82 \%)$ of the countries around the world utilize a range of names, terms, or phrases (NTP's) to describe broader impacts. Broader impacts-like NTP's were originally identified based on three overarching features.

The first feature was that the NTP had to be focused on achieving something societally desirable. The second feature was that it had to encompass a process function. The third feature was that it needed to encourage achieving a specific goal ${ }^{[1]}$. Examples of these NTP's are found in Figure 1 (Fig.1) and organized by country, except for the European Unions (EU's) Responsible Research and Innovation (RRI) and the Research Excellence Framework (REF).

Given that countries have their own unique words that can be equivalent to broader impacts, broader impactslike NTPs could be also characterized as societal benefitting-like NTPs. This is a more accessible, politically correct, and universal classification system compared to categorizing these NTPs as broader impacts-like ${ }^{(\mathrm{CN} 1)}$. Note: CN stands for content notes and are located at the bottom of this article.

After the NTPs from around the world were identified, collected and organized, an NTP cross-case analysis was performed in 2014-2015 and again in 2016-2017. This was done to elucidate a definition of broader 


\begin{tabular}{|l|c|}
\hline Broader Impacts-Like Names, Terms, and Phrases & Location \\
\hline Valorization & United Kingdom \\
\hline Benefit to Society \& Economy and Value of Public Engagement & Canada \\
\hline Knowledge Mobilization & India \\
\hline Equity in Development & Africa \\
\hline Capacity Building & South America \\
\hline Harmonious Development & China \\
\hline National Economic \& Social Development and Social Influence & United States \\
\hline Ultimate Outcomes & European Union \\
\hline Responsible Research and Innovation and the Research Excellence Framework & \\
\hline
\end{tabular}

Figure 1: Broader Impacts-Like or Societal Benefitting-Like NTPs Aligned With Country and Entity

impacts using an established research methodology. This methodology is an extension of the previous work performed by Davis and Laas in 2013, which compared the European Union's (EU's) Responsible Research and Innovation (RRI) to NSF Broader Impacts (BI) ${ }^{[1]}$.

The definition resulting from this cross-case analysis is that broader impacts is a process involving people/stakeholders to achieve a societal benefit that is measured or assessed and is accomplished in a finite amount of time. This process can be done through one's teaching, research, service, and occupation. There can be broader impacts of almost anything. If done appropriately, broader impacts can lead to sustainable positive impacts [2], [3], [4], [5].

Stakeholders is not used here in a traditional imperialistic or colonized sense. The word stakeholders, is used to refer to almost anyone or anything involved in this process. Thus, all those involved in the process should be considered stakeholders and be treated equitably as partners and not subjects.

\section{Beginning to Understand the "Nature of Broader Impacts"}

This research-based definition enables one to better understand how to think in a broader impacts paradigm. This definition aligns with engagement frameworks and activities, including but not limited to: community engagement, engaged scholarship, outreach, and empowerment theory ${ }^{[6]}$, [7]. It also encompasses the aspects many researchers associate with NSF Broader Impacts (BI) [8], [9], [10].

The definition, and its similar basis to engagement theory provides the foundation for constructing a broader impacts conceptual framework $[6],[7],[8],[9]$. This new framework adheres to an engagement-outcome-impact 
paradigm (Fig. 2) that is societally-centric in the context of an individual's ${ }^{[11]}$ and group's developing identity and "living legacy" (CN2).

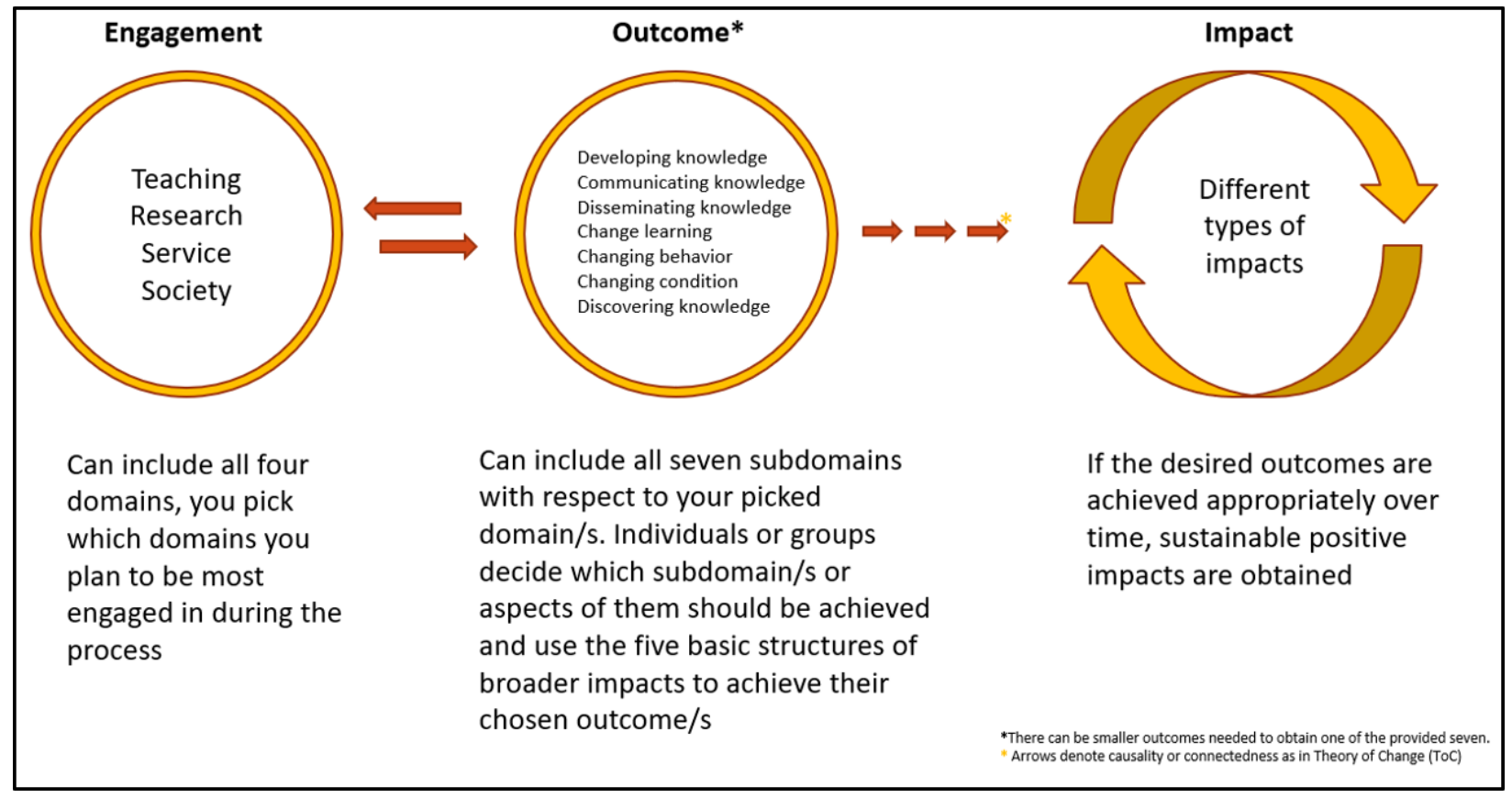

Figure 2: Engagement-Outcome-Impact Function of Broader Impacts

This thinking and the conceptual framework described below, provides insight into the unique "Nature of Broader Impacts" (CN3). The conceptual framework illustrates that broader impacts is a way of thinking, knowing, understanding, and acting.

Accepting the Nature of Broader Impacts (NBI) can help faculty and everyone in the academy move beyond a defensive response to a perceived agency requirement. This way of thinking, knowing, understanding, and acting helps all, meaning irrespective of discipline and research, teaching, service, or occupational focus, to acknowledge their role in positively impacting the present and the future.

\section{The Broader Impacts Conceptual Framework (BICF)}

In the Broader Impacts Conceptual Framework (BICF) there are four (4) major domains, each having seven (7) subdomains. The four major domains are research, teaching, service, and society. The central domain being society.

The seven subdomains found in each major domain are: i) developing knowledge; ii) communicating knowledge; iii) disseminating or sharing knowledge; iv) change learning; v) changing behavior; vi) changing condition; and vii) discovering knowledge. As we enter any one of the subdomains, we first have to ask: "Why have I selected this subdomain? What specific purpose and impact will this subdomain support?". Figure 3 (Fig 3.) provides an overview of the entire framework. 


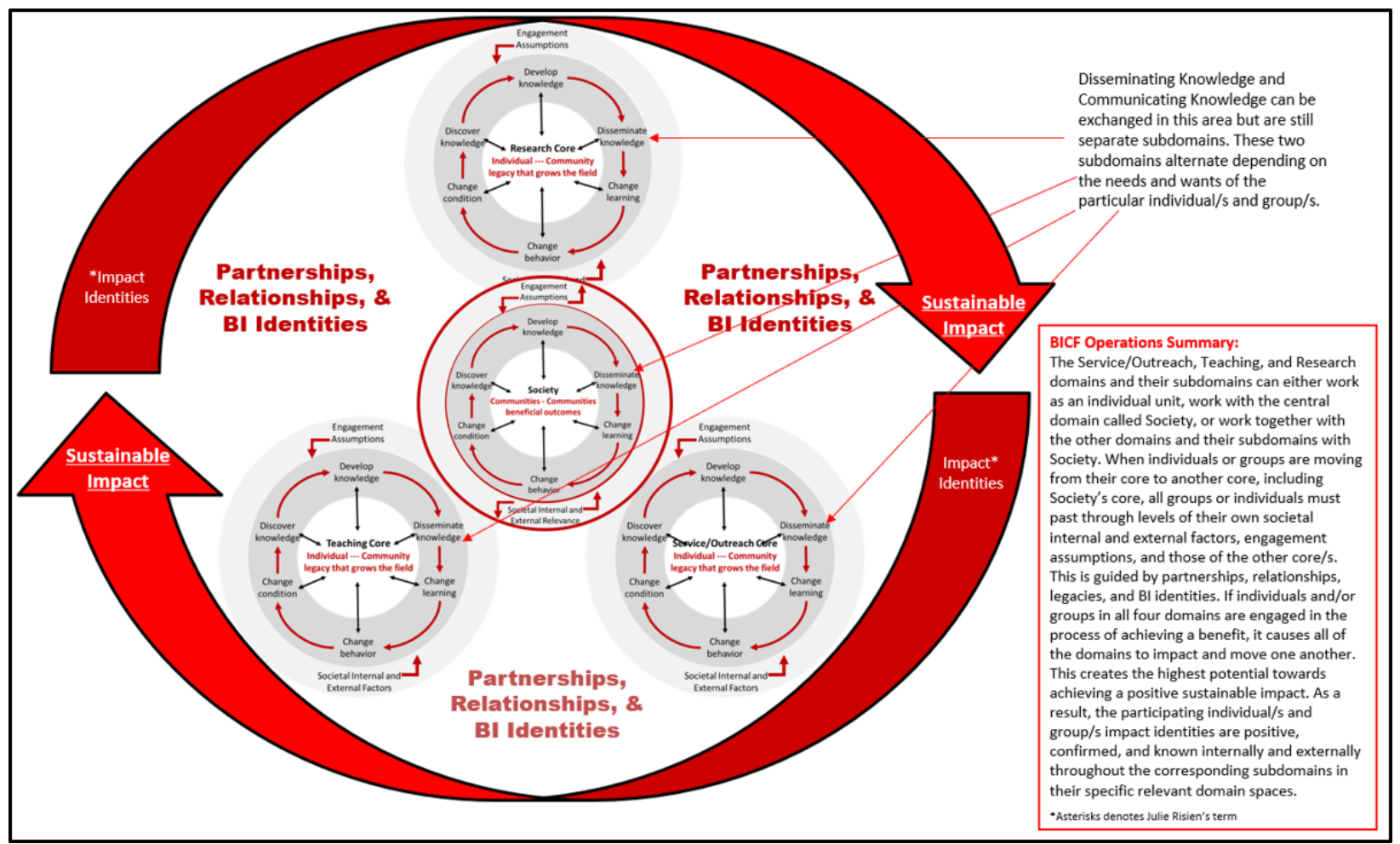

Figure 3: BICF Overview: The BICF is an Explicit Societal Centric Framework That Accounts For Engagement From Outside Into the Institution and Engagement From The Institution Into Society

While these questions must be considered for each subdomain, they also must be considered for each subdomain space. The subdomain space includes as well as represents smaller tasks, items, etc., which could be used, completed or done to accomplish an aspect of each respective subdomain.

Therefore, the subdomain space feature of the BICF suggests that there is an almost infinite set of activities that could be performed to accomplish any aspect of or a subdomain itself. This aligns with the perceived endless number of activities that could be used to accomplish a broader impact for NSF (CN4). This also includes the activities that could be done for any other type of foundation, agency, and organization with different broader impacts foci.

Both the major domains and their subdomains are all guided by an individual's or a group's professional/broader impacts identity and lead to the development of an individual's or group's impact identity(CN5). "Impact Identity/ies" was originally coined by Julie Risien at Oregon State University ${ }^{[12], ~[13] . ~}$

Within this framework, supporting each subdomain in their respective domain spaces are five basic structures. The five basic structures that undergird these subdomains and that are found in each major domain are: a) professional/broader impacts identity, which is the guiding principle for the five basic structures; b) outcomes; c) outputs, d) activities; and e) inputs [14], [15]. Figure 4 (Fig. 4) shows these 5 basic structures in the context of the BICF. Note: These "Five (5) Basic Structures of Broader Impacts" are also used to write a broader impacts narrative section in an NSF proposal. This will be discussed at another time.

When all of this is combined with engagement assumptions, internal and external relevance parameters, and legacy, the framework inherently acts like a Theory of Change (ToC). The inherent ToC nature of this 


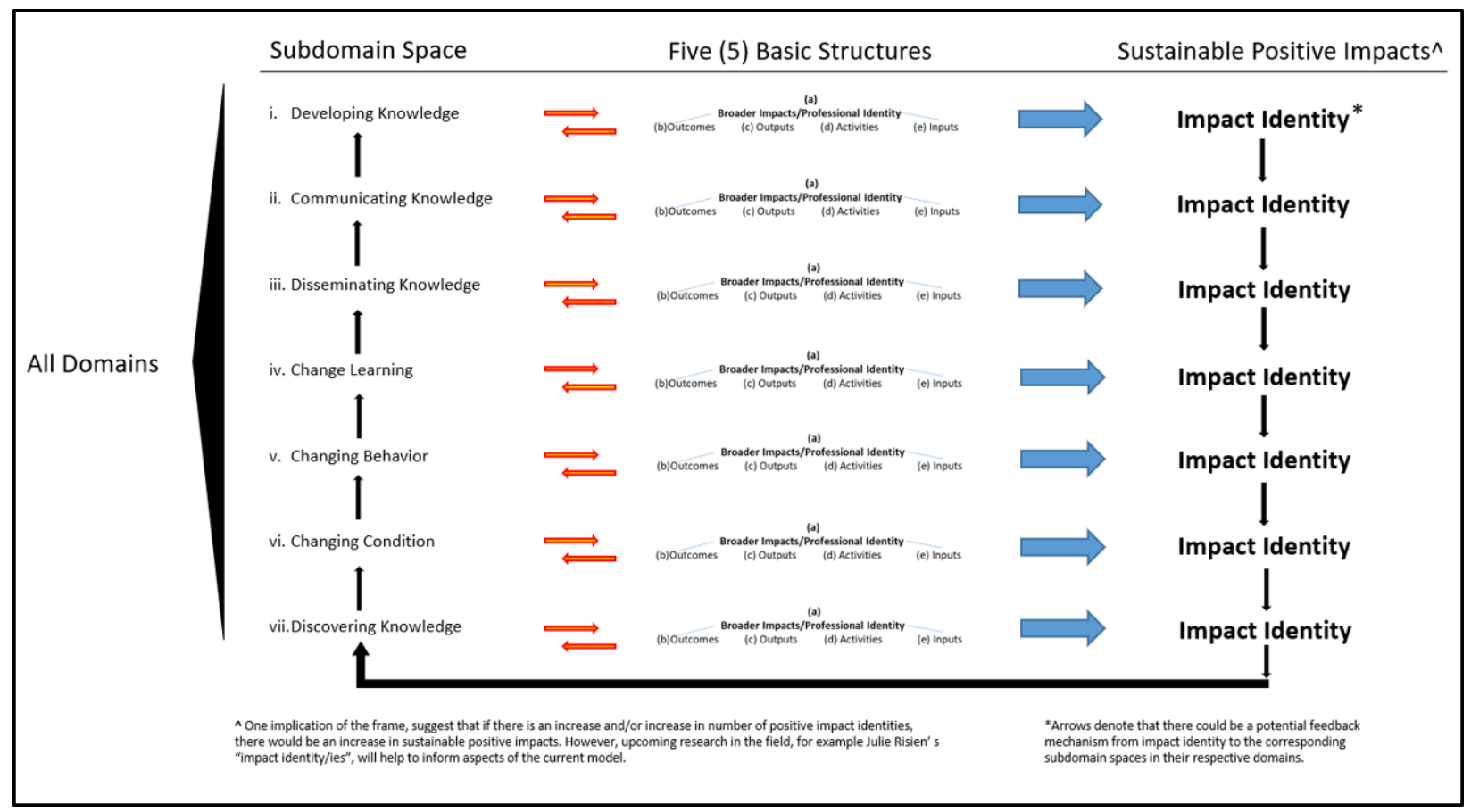

Figure 4: The Five (5) Basic Structures In the Context of the Domains, Subdomains, Subdomain Spaces, and Impact Identities as It Relates to Sustainable Positive Impacts

framework can help researchers see or develop mechanisms for facilitating sustainable positive impacts and create a positive impact identity. Inevitably, broader impacts should lead to sustainable positive impacts for society, which is the idea that NSF is trying to achieve through its BI 1-9 desired societal outcomes ${ }^{[16], ~[17] . ~}$

\section{A Critical Step}

Communicating knowledge, one of the seven subdomains, is a critical and major rate-determining step to effectively move through all four domains, move through the remaining corresponding subdomains, and move toward the development of sustainable positive impacts. In other words, communication and communicating knowledge are key.

Your ability to communicate and relate to different audiences depends on knowing who you are and how you plan to engage and benefit others through a living legacy in the context of your teaching, research, service, and occupation. This also means you should start to reflect, think about, and begin to answer the following question: What is your professional/broader impacts identity?

\section{Recommendations}

When you come into the University, first begin to reflect on your professional/broader impacts identity in the context of how you want to communicate your work. Second, select the individuals and groups in the 
public and in your field that align with your work and passions. Third, seek available training to improve your communication skills.

For those in the Science, Technology, Engineering, and Mathematics (STEM), Social Science, and STEM Education fields, this audience embodies your Science Communication (Sci-COMM) Ecosystem. For those of you who are not in these areas, consider it your Communication (COMM) Ecosystem. Sci-COMM Ecosystem is a phrase and model that was coined and developed by Melanie Harrison Okoro, PhD. In my opinion, it is one of the best upcoming science communication models under development that is relevant for today's knowledge abundance economy.

The members of your Sci-COMM Ecosystem or COMM-Ecosystem are called your stakeholders. Make sure to think about, treat with respect, communicate with, and listen to their ideas, suggestions, and advice. Those who share your Ecosystem space (educators, students, funding agencies, NGOs, colleagues, underrepresented ethnic groups, etc.) will help you identify the ideas that matter to society. Their input also will help you refine your professional/broader impacts identity and create a powerful broader impacts program or portfolio for your NSF-funded work.

\section{References}

[1] Davis M. and Laas K. (2013). "Broader Impacts" or "Responsible Research and Innovation"? A Comparison of Two Criteria for Funding Research in Science and Engineering. Science and Engineering Ethics, DOI 10.1007/s11948-013-9480-1.

[2]. Adetunji, O. and Renoe, S. (2017). Assessing Broader Impacts. MRS Advances, 1-6. Doi: 10.1557/ adv.2017.136.

[3]. Adetunji, O. and Thompson, M. (2016). The Broader Impacts Conceptual Framework (BICF) 2014 Lexicon Modification for the Brown University Engaged Scholarship and Broader Impacts Joint Committee Year End Report of 2015-2016. Brown University.

[4]. Thompson, M. (2014). The Broader Impacts in Research (BIR) Organization. BI Definitions Guide: An abbreviated collection of explanations that begins to provide a common language when discussing, practicing, understanding, and better articulating the dimensions of broader impacts (BI). [Brochure]. [Norman, Oklahoma].

[5]. Thompson, M. (2014). The Broader Impacts in Research (BIR) Organization. The Broader Impacts Conceptual Framework (BICF) Lexicon. The University of Oklahoma. http://bir.ou.edu/content/ broader-impacts-conceptual-framework-bicf.

[6]. Franz, N. (2009). A Holistic model of engaged scholarship telling the story across higher education's Missions. Journal of Higher Education Outreach and Engagement, Volume 13, number 4, p. 31- 50.

[7]. Franz, N. (2016). The Legacy and Future of a Model for Engaged Scholarship: Supporting a Broader Range of Scholarship. Journal of Higher Education Outreach and Engagement, Volume 20, Number 1, p. 217.

[8]. National Alliance for Broader Impacts (NABI). (2015). Broader Impacts Guiding Principles and Questions for National Science Foundation Proposals. [Tri-fold]. [Columbia, MO]: Adentunji, O., Coakley, C., Dawe, J., Dugan, C., Fields, J., Kobilka, S., Koroly, M., Menninger, H., Renoe, S., Ristvey, J., Scowcroft, G., Spohr, K., Sundararajan, S., Thompson, M., and Youngblood, T.

[9]. Nagy, D. (2016). Evaluating the Broader Impacts of Sponsored Research through the Lens of Engaged Scholarship, Journal of Research Administration. Vol. 47 Issue 1, p68-93. 
[10]. Verdín, D. (2017). Quantifying and Assessing Trends on the National Science Foundation's Broader Impact Criterion Paper presented at 2017 ASEE Annual Conference \& Exposition, Columbus, Ohio. https : $/ /$ peer. asee.org/28778.

[11]. Kail, R., and Cavanaugh. J. (2000). "Identity and Self-Development". Human Development: A Lifespan View, 2nd edition. Belmont, CA: Wadsworth.

[12]. Risien, J. (2015). Unveiling Identities and Enhancing Impacts. STEM-Fit Conference. Evanston, IL.

[13]. Risien, J. and Storksdieck, M. (2018). Unveiling Impact Identities: A Path for Connecting Science and Society. Integrative and Comparative Biology. in press.

[14]. College of Continuing Education, Division of Public and Community Services. (2007). Framework for the Mid-Continent Comprehensive Center (MC3), an Educational Technical Assistance Center, Planning Documentation. The University of Oklahoma.

[15]. Thompson, M. (2018). The Broader Impacts in Research (BIR) Organization. Faculty Success: A brief description of our research-based scholarly approach to practice in assisting faculty with broader impacts on their National Science Foundation (NSF) proposals and how faculty who receive this assistance perform compared to their peers. [Brochure]. [Norman, Oklahoma]. Electronic details: http: //bir.ou.edu/content/evaluating-return-investment-roi.

[16]. National Science Foundation (NSF). (2018). Proposal \& Awards Policies and Procedures Guide (PAPPG) 18-1. OMB Control Number 3145-0058, p. 45, Chapter II-12.

[17]. National Science Foundation. (2012). NSF at a glance. http://www.nsf .gov/about/glance.jsp. Accessed May 1, 2018.

\section{Content Notes (CN)}

CN1. Currently we have not been able to investigate as to whether this phenomenon has occurred in the Middle East.

CN2. Living legacy implies how you are known while living and is not in terms of death.

CN3. The "Nature of Broader Impacts" refers to key principles and ideas which provide a description, understanding, and the action of broader impacts as a way of knowing. It also applies to the characteristics of broader impacts knowledge.

CN4. There are some features of the BICF which limit what activities are plausible and would provide a greater societal benefit. This suggests that there are broader impacts quality levels.

CN5. In the BICF, professional/broader impacts identity is based on the research-based definition of broader impacts. According to this definition, broader impacts must be done with stakeholders, measurable, time dependent, positive, and societally beneficial. Impacts are not required to have any of these aspects. Therefore, there is a distinction between professional/broader impacts identity and impact identity in the BICF. These are two related but distinct concepts. This will be discussed in detail at another time. 